\title{
Diagnóstico qualitativo da poluição atmosférica em Rio Grande-RS, 2000 a 2002
}

\author{
Michelle Rodrigues Nóbrega* \\ Nisia Krusche*
}

\section{Resumo}

A poluição atmosférica e suas possíveis implicações sobre a saúde são analisadas neste trabalho para realizar um diagnóstico qualitativo para a região de Rio Grande, RS, no período de 2000 a 2002. Os dados utilizados foram: a percepção da população quanto à poluição do ar, internações por doenças respiratórias, níveis de concentração de poluentes, e condições meteorológicas. Todas as análises apresentaram como fator de distribuição as diferentes estações do ano. A associação entre níveis de concentração de poluentes, internações por doenças respiratórias e variáveis meteorológicas revelou correlação significativa. Este estudo do comportamento das baixas hospitalares por doenças respiratórias, revelou algumas associações, tais como: proximidade dos bairros com as indústrias; maior freqüência em crianças menores de cinco anos; e, entre níveis de poluentes do ar, internações por doenças e condições meteorológicas.

Palavras-chave: Poluição do ar; Poluentes do ar; Doenças respiratórias; Concentração máxima de poluentes.

\footnotetext{
* Doutoranda em Educação - Universidade Federal de Pelotas (nobregarm@terra.com.br).

** Professora Dra. - Núcleo de Física Ambiental - Centro de Ciências Computacionais - Universidade Federal do Rio Grande (nkrusche@furg.br)
}

Geosul, Florianópolis, v. 25, n. 50, p 129-150, jul./dez. 2010 
NÓBREGA, M.R \& KRUSCHE, N. Diagnóstico qualitativo da poluição ...

Qualitative diagnosis of the atmospheric pollution in Rio GrandeRS, from 2000 to 2002

\begin{abstract}
The atmospheric pollution and its implications to health are analyzed to establish a qualitative diagnosis for region of Rio Grande, RS, from 2000 to 2002. The data consists of an evaluation of the perception of the population about air pollution, number of internments due to the respiratory diseases, level of pollutants, and weather systems. All the analyses were performed taking into account the different seasons of the year. The association among levels of pollution concentration and the internments due to respiratory disease revealed a significant correlation. This study of the behavior of low hospital for respiratory tract diseases, showed some associations, such as: proximity to neighborhoods with the industries; often in children under 5 years; and between levels of air pollutants, internments for diseases and weather conditions.
\end{abstract}

Key words: Air pollution; Air pollutants; Respiratory tract diseases; Maximum concentration.

\title{
Introdução
}

A poluição do ar associada às internações por doenças respiratórias vem sendo estudada por vários pesquisadores em nível nacional e internacional. O material particulado costuma ser associado como fator de influência no total de mortes em idosos e crianças, internações e mortes por doenças cardiovasculares e respiratórias (BRAGA, 1998; SALDIVA et al, 1994; SALDIVA et al, 1995). Os poluentes que causam inflamação são muitos, sendo que entre os mais comuns está o material particulado e os oxidantes fotoquímicos (por exemplo, ozônio - $\mathrm{O}_{3}$ ). Indivíduos submetidos a episódios de poluição, com reações inflamatórias, acabam sendo alvos de infecções. 
NÓBREGA, M.R \& KRUSCHE, N. Diagnóstico qualitativo da poluição ...

O grupo etário populacional mais suscetível aos efeitos da poluição, de forma geral, são as crianças, por consumirem, proporcionalmente, mais ar do que os adultos. Outro fator que contribui para esta exposição maior é o seu sistema imunológico menos desenvolvido. Crianças têm ainda mais contato com ambientes externos, onde correm maiores riscos de contaminação por agentes químicos (BRAGA, 1998).

A região estuarina da Lagoa dos Patos tem sido objeto de pesquisas sobre contaminações atmosféricas, devido a vários tipos de poluentes, com abordagens distintas.

Vanz, Mirlean e Baisch (2003) avaliaram a poluição do ambiente pelo componente chumbo na cidade de Rio Grande, onde foi verificado que existe um processo de contaminação atmosférica pelo mesmo, o que não permitiu, todavia, classificá-lo como originário das fontes industriais e sim, decorrente de atividades ligadas à pesca artesanal, ou ainda, outras atividades não identificadas realizadas pelo grupo populacional. Sobretudo, apontaram que há fortes indicativos que os limites legais da qualidade do ar, em relação ao chumbo, sejam ultrapassados em decorrência da associação de alto teor desse metal associado à elevada concentração de material particulado atmosférico.

Vários trabalhos já abordaram o impacto de poluentes no ar que respiramos. Mirlean et al (2002) constataram que as emissões das fábricas de fertilizantes enriquecem o ambiente com fluoreto dissolvido na água da chuva.

Já Saraiva e Krusche (2002), constataram que a análise sazonal das variáveis atmosféricas, predominantes durante as ocorrências de episódios com elevada concentração de Particulado Total em Suspensão (PTS), revelou comportamento diferenciado para cada estação do ano. Isso indica que diferentes sistemas de tempo ocorreram durante as concentrações de PTS para os anos de 1990, 1994, 1997, 1998 e 1999.

Bortolini e Krusche (1998), realizaram um estudo do comportamento das reuniões dos poluentes PTS e $\mathrm{SO}_{2}$ frente aos parâmetros velocidade do vento e precipitação, onde foi 
NÓBREGA, M.R \& KRUSCHE, N. Diagnóstico qualitativo da poluição ...

evidenciado que as direções do vento predominantes nos eventos de maior concentração de $\mathrm{SO}_{2}$ foram sul e sudoeste.

Este trabalho apresenta uma caracterização da poluição do ar em Rio Grande, partindo da percepção da população quanto à contaminação do ar, através de entrevistas semi-estruturadas, distribuição mensal das internações por doenças respiratórias ocorridas no período de 2000 a 2002, e caracterização meteorológica dos episódios de máxima concentração de poluentes. Associa, também, os níveis de poluentes e baixas hospitalares por doenças respiratórias durante o ano de $2002^{1}$, enfatizando a faixa etária compreendida entre zero (0) e cinco (5) anos. Todas as análises realizadas tiveram como fator de distribuição as diferentes estações do ano.

\section{Metodologia}

O município de Rio Grande, região de estudo, compreende uma área de $3.338 .35 \mathrm{~km}^{2}$ (Figura 1). Localiza-se na região estuarina da Lagoa dos Patos, RS, com a configuração de uma restinga na planície costeira sul do Estado. Possui clima subtropical úmido (STRAHLER; STRAHLER, 1996).

As doenças do aparelho respiratório consideradas neste estudo foram: pneumonia, doença pulmonar obstrutiva crônica, sinusite, rinite, asma, bronquite, bronquiolite, insuficiência respiratória e tuberculose pulmonar. Associações significativas entre doenças respiratórias e níveis de poluentes foram verificadas em diversos estudos (SALDIVA et al, 1994; SALDIVA et al, 1995; BELL et al, 2005). Destaca-se, com base nessa citação, que este estudo tem o intuito de analisar as possíveis correlações entre as hospitalizações por doenças respiratórias e os episódios de poluição do ar. Mas, ressalta-se que em muitos casos a poluição do

1 Ressalta-se que somente o ano de 2002 foi utilizado para a análise, devido à indisponibilidade dos dados referentes ao monitoramento da qualidade do ar para 2000 e 2001. 
NÓBREGA, M.R \& KRUSCHE, N. Diagnóstico qualitativo da poluição ...

ar não é o único fator que influencia o aparecimento ou agravo de doenças respiratórias.

FIGURA 1: Localização da área de estudo, uma representação da região estuarina da Lagoa dos Patos, com a indicação da sede dos municípios de Rio Grande e São José do Norte, incluindo a localização das indústrias e das estações de monitoramento da FEPAM. O número 1 corresponde à estação CEEE, o 2 à estação Corsan, o 3 à estação Praça Montevideo, e o 4 à estação Cassino. As industrias de fertilizante estão caracterizadas pelo símbolo circulo, a refinaria por

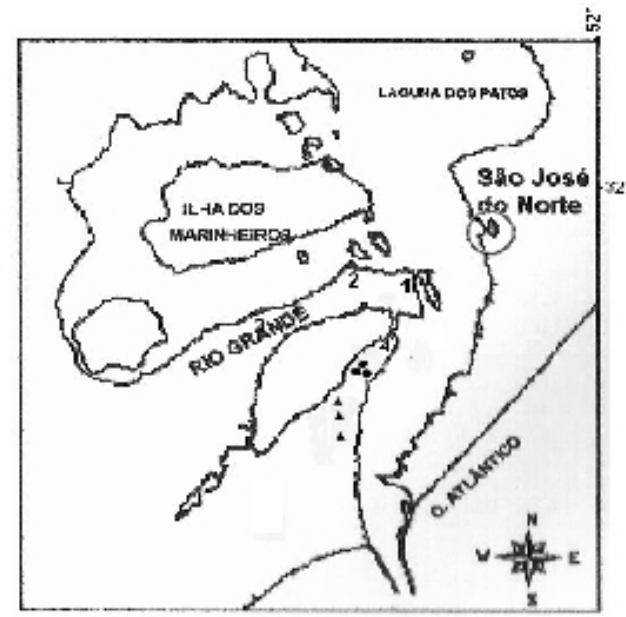
losângulo e as industrias de extração e refino de óleo vegetal por triângulo.

Utilizou-se, então, a análise do comportamento das internações por doenças respiratórias, por bairros, devido ao banco dados do Sistema Único de Saúde (SUS), apresentar na sua estrutura relacional de identificação do paciente, a classificação de cada registro de baixa hospitalar, constando a origem do bairro do paciente. Desta forma, a categoria bairro possibilitou localizarmos espacialmente a dinâmica das hospitalizações ao longo do período analisado neste estudo. As áreas do município de Rio Grande, selecionadas (Figura 2) para análise foram: os locais mais próximos às indústrias e os mais afastados pelas mesmas, ou seja, utilizou-se o critério da distância em relação às fontes estacionárias de poluentes para a seleção dos 20 locais estudados. Ressalta-se que foram selecionados 19 bairros da cidade do Rio Grande e o município de São José do Norte.

Foram realizadas 1000 entrevistas estruturadas semiabertas $^{2}, 50$ para cada um dos 20 locais. A realização das

2 As entrevistas questionaram: Existe poluição do ar em seu bairro? Caso fosse afirmativa a resposta, foi questionado se nos dias que percebiam que existia aumento na concentração de poluentes, sentiam: ardência nas narinas, falta de ar, rinite, sinusite, tosse, escamação da pele, dor 
NÓBREGA, M.R \& KRUSCHE, N. Diagnóstico qualitativo da poluição ...

entrevistas ocorreu em diversas épocas de 2001 e 2002, envolvendo as diferentes estações do ano. As entrevistas realizadas durante a primavera ocorreram em 2002 e as demais no ano de 2001. Os períodos de estudos junto aos bairros não obedeceram ao mesmo intervalo de tempo, cerca de $48 \%$ das entrevistas foram realizadas no verão, $24 \%$ foram realizadas na primavera, $11 \%$ no outono e $17 \%$ no inverno.

FIGURA 2: localização dos bairros e do município de São José do Norte em relação às indústrias. Os bairros mais próximos às indústrias estão hachurados de cinza claro, onde 1 corresponde a Getulio Vargas, 2-Santa Tereza, 3-Centro, 4- Navegantes, 5Cidade Nova, 6-Hidráulica, 7- Ernesto Buccholz, 8-Junção, 9-Bernadete e 10Barra. Os bairros mais afastados das indústrias estão sob hachura cinza escuro, onde 11 significa Santa Rosa, 12-Carreiros, 13-Jardim do Sol, 14-

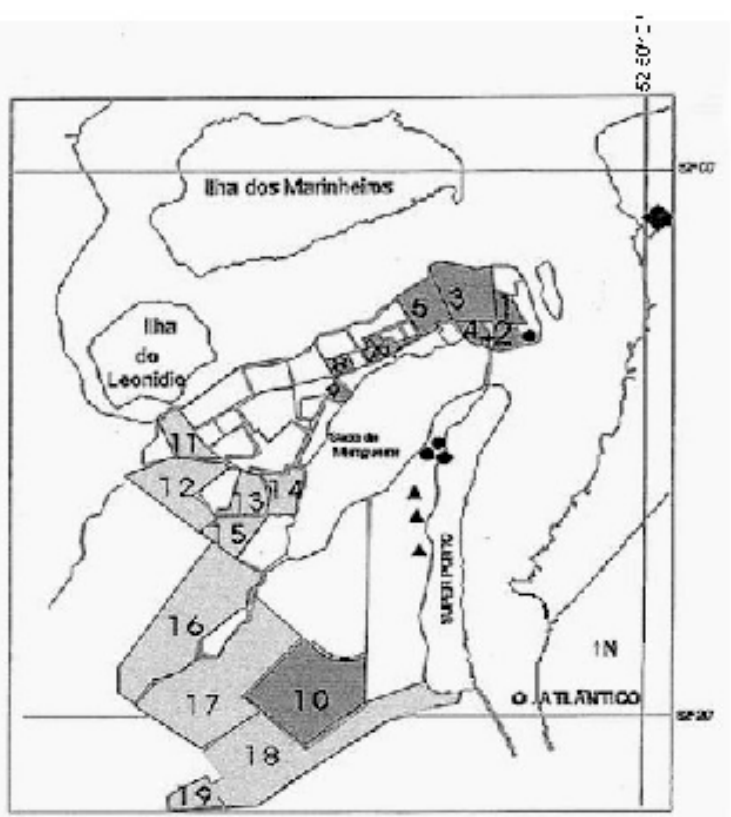
Trevo, 15-Parque São Pedro, 16-

Senandes, 17-Bolacha, 18-Cassino e 19-Querência. O município de São José do Norte corresponde ao losango grande preto. As indústrias de fertilizante estão caracterizadas pelo símbolo circulo a refinaria por losângulo e as indústrias de refino e extração de óleo vegetal por triângulo.

Os dados utilizados neste estudo referem-se às internações do SUS e foram fornecidos pelo Setor de Custos do Hospital

nos olhos, dor de cabeça, tontura, entupimento do nariz e perda dos sentidos. Essas categorias foram sistematizadas com base na literatura internacional e nacional a respeito da poluição do ar e seus efeitos sobre a saúde, dentre elas, cita-se a obra: American Thoracic Society. What constitutes an adverse health effect of air pollution? Official statement of the American Thoracic Society. Am J Respir Crit Care Med 2000;161(2):665-73. 
NÓBREGA, M.R \& KRUSCHE, N. Diagnóstico qualitativo da poluição ...

Universitário (HU) e Setor de Arquivos da Associação de Caridade Santa Casa de Misericórdia. Quanto à análise dos dados referentes às intervenções médicas por doenças respiratórias, optou-se por utilizar o período de três anos (2000 a 2002), uma vez que os dados utilizados compreendem as hospitalizações do SUS, onde o público alvo concentra-se em indivíduos com condições econômicas menos favorecidas, tornando-se mais suscetível às doenças do trato respiratório, principalmente crianças.

Os dados referentes aos níveis de componentes poluidores para o período de 2000 a 2002 foram solicitados junto a Fundação de Proteção Ambiental Henrique Roessler (FEPAM). Salienta-se que somente os Índices de Qualidade do $\mathrm{Ar}^{3}$ (IQArs) e as máximas concentrações foram fornecidas apenas para o ano de 2002 e sob a condição de um relatório em formato digital fornecido pela instituição. Foram utilizados também dados sobre concentração de poluentes e monitoramento dos mesmos, dispostos no site da instituição referida durante o período analisado por esta investigação.

Foi realizada, após a identificação dos episódios de poluição do ar para o ano de 2002, a análise global dos dados meteorológicos para o total dos episódios, e análise sazonal deste conjunto de episódios, onde foram utilizados dados médios diários de pressão, temperatura, taxa de precipitação, umidade relativa, intensidade da velocidade do vento, medidas pela Estação Meteorológica de Rio Grande. O valor médio diário de cada variável foi descontado das Normais Climatológicas provisórias de Rio Grande para o período de 1991 a 2000 (KRUSCHE et al, 2002), de acordo com o mês de ocorrência da mesma. No segundo momento, foi analisado o comportamento das variáveis

${ }^{3}$ O Índice de Qualidade do Ar proposto pela FEPAM consiste em uma ferramenta utilizada para decompor as concentrações medidas de diversos poluentes em um único valor adimensional, que permite a comparação com os limites legais de concentrações para os poluentes monitorados. 
NÓBREGA, M.R \& KRUSCHE, N. Diagnóstico qualitativo da poluição ...

meteorológicas para os episódios de máxima concentração de PTS e $\mathrm{O}_{3}$, considerando os quatro dias que antecedem os episódios.

\section{Resultados e discussão}

\section{Percepção da população quanto à poluição do ar}

Quase metade dos entrevistados relatou sentir os efeitos adversos sobre a saúde devido à poluição atmosférica. Dentre o total de entrevistados (1000), apenas $9 \%$ declarou ser fumante, ou seja, 90 pessoas. Cerca de $4 \%$ dos entrevistados aferiram sentir a contaminação através de aspectos físicos, como, por exemplo, nuvens de poeira, etc. Nos bairros mais próximos às fontes de poluição, cerca de 54\% (270) dos moradores entrevistados relataram que percebem os sintomas possíveis de estarem associados à poluição do ar. Nestes bairros, os sintomas mais relatados foram: entupimento das narinas (50\%), dor de cabeça (49\%) e ardências nas narinas (49\%). Já os moradores entrevistados dos bairros mais afastados, 31\% (155) relataram sentir os efeitos da poluição do ar sob a saúde. Sendo que os sintomas mais relatados pelos moradores foram: rinite (16\%), entupimento do nariz $(13 \%)$, tosse $(12 \%)$, ardência nas narinas (11\%), sinusite (11\%), dor de cabeça (11\%) e falta de ar (11\%).

Apesar de terem sido realizadas apenas 170 entrevistas, número bem inferior, por exemplo, ao verão, quando foram realizadas 477 entrevistas, o inverno foi o período em que a população referiu-se mais a alguns sintomas, ou seja, 98 registros de reclamações apontaram o sintoma entupimento das narinas, e 74 registros aferiram aos sintomas tosse e dor de cabeça, enquanto efeitos que possam estar associados a episódios de poluição do ar. 
NÓBREGA, M.R \& KRUSCHE, N. Diagnóstico qualitativo da poluição ...

Distribuição sazonal das internações por doenças respiratórias segundo distância das fontes de poluição do ar de 2000 a 2002

Em 2000, as doenças respiratórias foram responsáveis por $24 \%$ das hospitalizações na cidade. Em 2001, 30\% das baixas hospitalares na cidade foram referentes a esse tipo de doença. A maior ocorrência, dentre o período analisado, ocorreu no ano de 2002, registrando $34 \%$ total de internações.

Em 2000, números expressivos de baixas, inclusive por crianças menores que cinco anos, ocorreram no outono e no inverno (conforme Quadro 1). Durante o ano de 2001, os meses que registraram mais hospitalizações, concentraram-se na primavera e no inverno. Nesse ano, números mais elevados de baixas por crianças menores que cinco anos situaram-se no outono e no inverno. No ano de 2002, os meses onde se registrou internações mais elevadas, situaram-se na primavera, inverno e verão. A análise do comportamento das baixas hospitalares por crianças com idade inferior a cinco anos, revelou maior número de internações no inverno e outono. Ressalta-se que um número expressivo de registros dessas baixas para essa faixa etária ocorreu no inverno, em 2000, 2001 e 2002.

\begin{tabular}{|c|c|c|c|c|c|c|}
\hline \multirow{3}{*}{ MESES } & \multicolumn{6}{|c|}{ ANOS } \\
\hline & \multicolumn{2}{|c|}{2000} & \multicolumn{2}{|c|}{2001} & \multicolumn{2}{|c|}{2002} \\
\hline & $\begin{array}{c}\text { TOTAL } \\
\text { MENSAL }\end{array}$ & $\begin{array}{l}\text { Cijanças } 5 \\
\text { que } 5 \text { anos }\end{array}$ & $\begin{array}{c}\text { TOTAL } \\
\text { MENSAL }\end{array}$ & $\begin{array}{l}\text { Crianças }= \\
\text { que } 5 \text { anos }\end{array}$ & $\begin{array}{l}\text { TOTAL } \\
\text { MENSAL }\end{array}$ & $\begin{array}{l}\text { Crianças }= \\
\text { que } 5 \text { anos }\end{array}$ \\
\hline 1 & 68 & 17 & 114 & 24 & 160 & 39 \\
\hline 2 & 98 & 46 & 106 & 39 & 111 & 23 \\
\hline 3 & 145 & 88 & 146 & 67 & 135 & 68 \\
\hline 4 & 127 & 49 & 167 & 83 & 164 & 41 \\
\hline 5 & 148 & 48 & 114 & 89 & 194 & 101 \\
\hline 6 & 136 & 50 & 148 & 55 & 200 & 96 \\
\hline 7 & 175 & 63 & 172 & 51 & 174 & 54 \\
\hline 8 & 188 & 77 & 212 & 67 & 151 & 32 \\
\hline 9 & 127 & 48 & 215 & 78 & 184 & 56 \\
\hline 10 & 123 & 43 & 200 & 77 & 256 & 70 \\
\hline 11 & $10 \theta$ & 34 & 171 & 41 & 158 & 62 \\
\hline 12 & 59 & 39 & 85 & 27 & 407 & 64 \\
\hline TOTALIANO & 1504 & 602 & 1850 & 698 & 2294 & 706 \\
\hline
\end{tabular}

QUADRO 1: Número de internações por doenças respiratórias total e por crianças menores que cinco anos, mensal e anual de 2000 a 2002. 
NÓBREGA, M.R \& KRUSCHE, N. Diagnóstico qualitativo da poluição ...

A distribuição espacial sazonal das internações por enfermidades respiratórias, segundo o critério distancia das fontes, constatou que:

a) No período de 2000 a 2002, 46\% (692), 34\% (629) e 41\% (940), respectivamente, foram internações de moradores próximos às fontes de poluição do ar. Em 2000, os maiores percentuais de hospitalizações ocorreram no outono e inverno, sendo que esses coincidem também com os períodos aos quais mais crianças foram internadas, devido a doenças respiratórias, principalmente nos meses março e agosto, os quais, juntos, totalizam aproximadamente 39\% das internações ocorridas nestes bairros. Em 2001, as intervenções médicas distribuíramse ao longo dos dez bairros de forma irregular e descontínua ${ }^{4}$. Observaram-se, também, números mais elevados de baixas hospitalares no inverno e no outono, nos meses de maio e setembro, sendo $25 \%$ das internações da faixa etária menor que cinco anos. Em ano 2002, percentuais significativos concentraram-se no inverno e na primavera, nos meses junho e julho, totalizando $27 \%$ das baixas para este conjunto de bairros dentro da faixa etária menor que cinco anos.

b) Para o conjunto de bairros mais afastados das fontes de poluição do ar, a distribuição do número de hospitalizações por doenças respiratórias ao longo do período analisado revelou que:

4 Ressalta-se que, em algumas localidades, como por exemplo, o Jardim do Sol, com condições sócio-econômicas mais elevadas, a ausência de internações ou a inexpressível ocorrência, oriunda destes, justifica-se devido aos residentes buscarem atendimento através da rede de atendimento hospitalar particular. Sendo assim, como os dados utilizados neste estudo abrangem o Sistema Único de Saúde (SUS), internações particulares e por meio de convênios não constam nos dados de análise. 
NÓBREGA, M.R \& KRUSCHE, N. Diagnóstico qualitativo da poluição ...

1. Tanto em 2000 como em 2001, $10 \%$ do total de internações — ou seja, 150 e 180 respectivamente foram de moradores destes bairros, sendo que $80 \%$ do total de baixas ocorridas nestes bairros foram de crianças menores de cinco anos. Em 2002, 12\% (229) do total de hospitalizações foram de moradores desse conjunto de bairro, e cerca de $90 \%$ destas foram de crianças menores que cinco anos. Tanto no ano de 2000, como em 2001, ocorreram intervenções médicas mais expressivas no outono e no inverno. Para o ano de 2002 verificam-se números elevados no inverno e na primavera.

2. Verificou-se que o número de internações por bairro em crianças menores que cinco anos, ao longo do período em análise, encontra-se em ascensão, para a maioria dos bairros analisados neste estudo.

\section{Níveis de poluentes $\left(\mathrm{PTS}, \mathrm{O}_{3}\right.$.), condições meteorológicas e internações por doenças respiratórias em 2002}

Segundo dados da FEPAM $^{5}$, a concentração de PTS ultrapassou o padrão secundário de qualidade do $\operatorname{ar}^{6}$ apenas uma única vez e em uma única estação de monitoramento, durante o ano de 2002. Houve, no entanto, 14 episódios de IQAr (Índice da Qualidade do Ar) regular para PTS no ano de 2002, conforme pode ser observado na Tabela 1. Ocorreram 13 episódios de IQAr regular para o poluente ozônio, onde se registraram concentrações superiores a $80 \square \operatorname{gm} 3$ (Tabela 2).

\footnotetext{
${ }^{5}$ Fonte de obtenção dos dados já mencionada no item metodologia.

${ }^{6}$ São concentrações de poluentes abaixo das quais se prevê o mínimo efeito adverso sobre o bem-estar da população, assim como o mínimo dano à fauna, flora, aos bens materiais e ao meio ambiente em geral.
} 
NÓBREGA, M.R \& KRUSCHE, N. Diagnóstico qualitativo da poluição ...

TABELA 1: Dias em que ocorreram concentrações de PTS $>50 \mu \mathrm{g} / \mathrm{m}^{3}$, IQARs e a respectiva estação para cada mês do ano de 2002

\begin{tabular}{|c|c|c|c|c|}
\hline \multirow[b]{2}{*}{ Meses } & \multicolumn{4}{|c|}{ ESTAÇŌES } \\
\hline & RGCE & $\mathrm{RGCO}$ & RGPRIM & RGRC \\
\hline 1 & $x$ & $15-10 A \mathrm{~s} 63 \mu \mathrm{g} / \mathrm{m}^{\mathrm{x}}$ & $x$ & - \\
\hline 2 & $02-10 \mathrm{Ar}, 62 \mu \mathrm{g} \mathrm{mm}^{\prime}$ & $26-10 \mathrm{~A} \cdot 55 \mu \mathrm{g} / \mathrm{m}^{\prime}$ & $x$ & - \\
\hline 3 & $x$ & $x$ & - & - \\
\hline 4 & $\mathrm{x}$ & -- & $\mathrm{x}$ & - \\
\hline 5 & 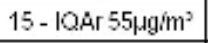 & $27-10 \mathrm{Ar} 72 \mu \mathrm{g} / \mathrm{m}^{2}$ & $15-10 A r 52 \mu g^{\prime} m^{2}$ & - \\
\hline 6 & 14 - ІОAि $53 \mu g m^{2}$ & $\mathrm{x}$ & $\mathrm{x}$ & $x$ \\
\hline 7 & $02-$ OA. $51 \mu \mathrm{gim} \mathrm{m}^{2}$ & $\mathrm{x}$ & $\mathrm{x}$ & 27 - IOAr 65 $\mu g y^{\prime} m^{2}$ \\
\hline 8 & $x$ & $x$ & $x$ & $01-10 \mathrm{Ar} 73 \mu \mathrm{g} / \mathrm{m}$ \\
\hline 9 & 06 - 1OAि 56hg $/ \mathrm{m}^{2}$ & $06-10 A r 54 \mu \mathrm{g} / \mathrm{m}^{2}$ & 06 - IOAि $52 \mu \mathrm{g}^{\prime} \mathrm{m}^{2}$ & $24-10 \Delta \mathrm{Ar} 51 \mu \mathrm{g} / \mathrm{m}^{2}$ \\
\hline 10 & 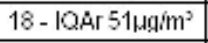 & $18-10 A r 54$ agim & $x$ & $x$ \\
\hline 11 & $\mathrm{X}$ & $11-$ lOAr 53ugim² & $\mathrm{x}$ & $x$ \\
\hline 12 & $x$ & $\mathrm{x}$ & $\mathrm{x}$ & $x$ \\
\hline
\end{tabular}

Fonte: Dados da Fundação Estadual de Proteção Ambiental Henrique Roessler/RS

Nota: Onde X: Ausência de monitoramento

\begin{tabular}{|c|c|c|}
\hline \multirow[b]{2}{*}{ Dlas de ocorrěncla } & \multicolumn{2}{|c|}{ ESTAÇŌEs } \\
\hline & RloGrande'IMUSEU móvel & RloGrandesCEEE mável \\
\hline 2806 & $86,03 \mu g^{\prime} \mathrm{m}^{2}$ & $\mathrm{x}$ \\
\hline 0808 & $81,07 \mu g^{\prime} \mathrm{m}^{2}$ & $x$ \\
\hline 21,08 & $x$ & $81,71 \mu \mathrm{gim} 2$ \\
\hline 25,08 & $x$ & $107,30 \mu \mathrm{g}^{\mathrm{m}} \mathrm{m}^{2}$ \\
\hline 23.08 & $\mathrm{x}$ & $88,14 \mu \mathrm{gin} x$ \\
\hline 2808 & $\mathrm{x}$ & $82,31 \mu \mathrm{gin} x$ \\
\hline 2709 & $\mathrm{x}$ & $81,83 \mu \mathrm{gim} \mathrm{m}^{2}$ \\
\hline 2809 & $\mathrm{x}$ & $94,53 \mu g i m^{x}$ \\
\hline $06 / 10$ & $\mathrm{x}$ & $100,10 \mu \mathrm{gg} / \mathrm{m}^{3}$ \\
\hline $07 / 10$ & $x$ & $92,50 \mu \mathrm{gim} \mathrm{m}^{2}$ \\
\hline $03 / 11$ & $\mathrm{x}$ & $83,15 \mu g i \gamma^{x}$ \\
\hline $13 / 11$ & $\mathrm{x}$ & $92,05 \mu g i m^{x}$ \\
\hline $14 / 11$ & $\mathrm{x}$ & $92,43 \mu g i m x$ \\
\hline
\end{tabular}

QUADRO 2: Dias em que ocorreram concentrações de $\mathrm{O}_{3}>80 \mu \mathrm{g} / \mathrm{m}^{3}$ e a respectiva estação para dia de ocorrência mensal do ano de 2002

Fonte: Dados da Fundação Estadual de Proteção Ambiental Henrique Roessler/RS

Nota: Onde X: Ausência de monitoramento

A análise sazonal do total de episódios ocorridos em 2002 evidencia que, $80 \%$ destes, distribuem-se ao longo do inverno e da primavera. Para o poluente $\mathrm{O}_{3}$, a maior incidência ocorreu na primavera $(54 \%)$ e no inverno $(46 \%)$, para o PTS, os maiores 
NÓBREGA, M.R \& KRUSCHE, N. Diagnóstico qualitativo da poluição ...

registros de episódios ocorreram no inverno (38\%) e na primavera $(30 \%)$.

Além do total de episódios referenciados acima, foram analisados, também, os dias de ocorrência de máxima concentração dos poluentes, $\mathrm{O}_{3}$ e PTS, conforme evidencia-se no Quadro 3.

\begin{tabular}{|c|c|c|c|c|}
\hline Estação & Sigla & Dia & Poluente & Concentração \\
\hline Rio GrandefPraça Montevideo & RGPRM & $15 / 05$ & PTS & $90 \mu \mathrm{g} / \mathrm{m}^{3}$ \\
\hline Rio Grande/Corsan & RGCO & $27 / 05$ & PTS & $150 \mu \mathrm{g} / \mathrm{m}^{: 5}$ \\
\hline Rio Grande/Cassino & RGRC & $12 / 08$ & PTS & $154 \mu g / m^{2}$ \\
\hline Rio Grande/CEEE & RGCE & $11 / 12$ & PTS & $124 \mu g^{\prime} \mathrm{m}^{3}$ \\
\hline Rio Grande/CEEE móvel & RGCE móvel & $25 / 08$ & $O_{3}$ & $107,3 \mathbf{\mu g} / \mathrm{m}^{3}$ \\
\hline
\end{tabular}

QUADRO 3: Máximas concentrações de poluentes, estação de monitoramento e dia de ocorrência. Fonte: Dados da Fundação

Estadual de Proteção Ambiental Henrique Roessler/RS

\section{Associação entre internações por doenças respiratórias e dias de máximas concentrações de poluentes}

No decorrer do ano de 2002, os maiores percentuais de internações referentes às doenças respiratórias centralizaram-se nos meses maio, junho, outubro e dezembro, registrando percentuais $8,4 \%, 8,8 \%, 11 \%$ e $18 \%$, respectivamente, em relação ao percentual total de baixas hospitalares. $53 \%$ das baixas nos bairros mais próximos das indústrias ocorreram entre os dias de máxima concentração e os cinco dias seguintes.

Foi constatado, no mês de maio, que os maiores números de hospitalizações ocorreram entre os dias de máxima concentração de PTS, 15 e 27 de maio, e os cincos dias posteriores. $49 \%$ das internações de crianças menores que cinco anos no mês de maio aconteceram entre os dias de ocorrência das máximas concentrações de PTS e os cincos dias subseqüentes (Figura 3).

Das 101 crianças internadas no mês de maio, quase metade dos casos, ou seja, 49 crianças, foram hospitalizadas no período descrito acima. 
NÓBREGA, M.R \& KRUSCHE, N. Diagnóstico qualitativo da poluição ...

No mês de agosto verificou-se que, entre o dia da máxima concentração para o PTS, 12 de agosto, e os cinco dias subseqüentes, ocorreram os maiores números de hospitalizações. Nesse período do mês, também ocorreram aproximadamente $46 \%$ das hospitalizações de crianças menores que 5 anos.

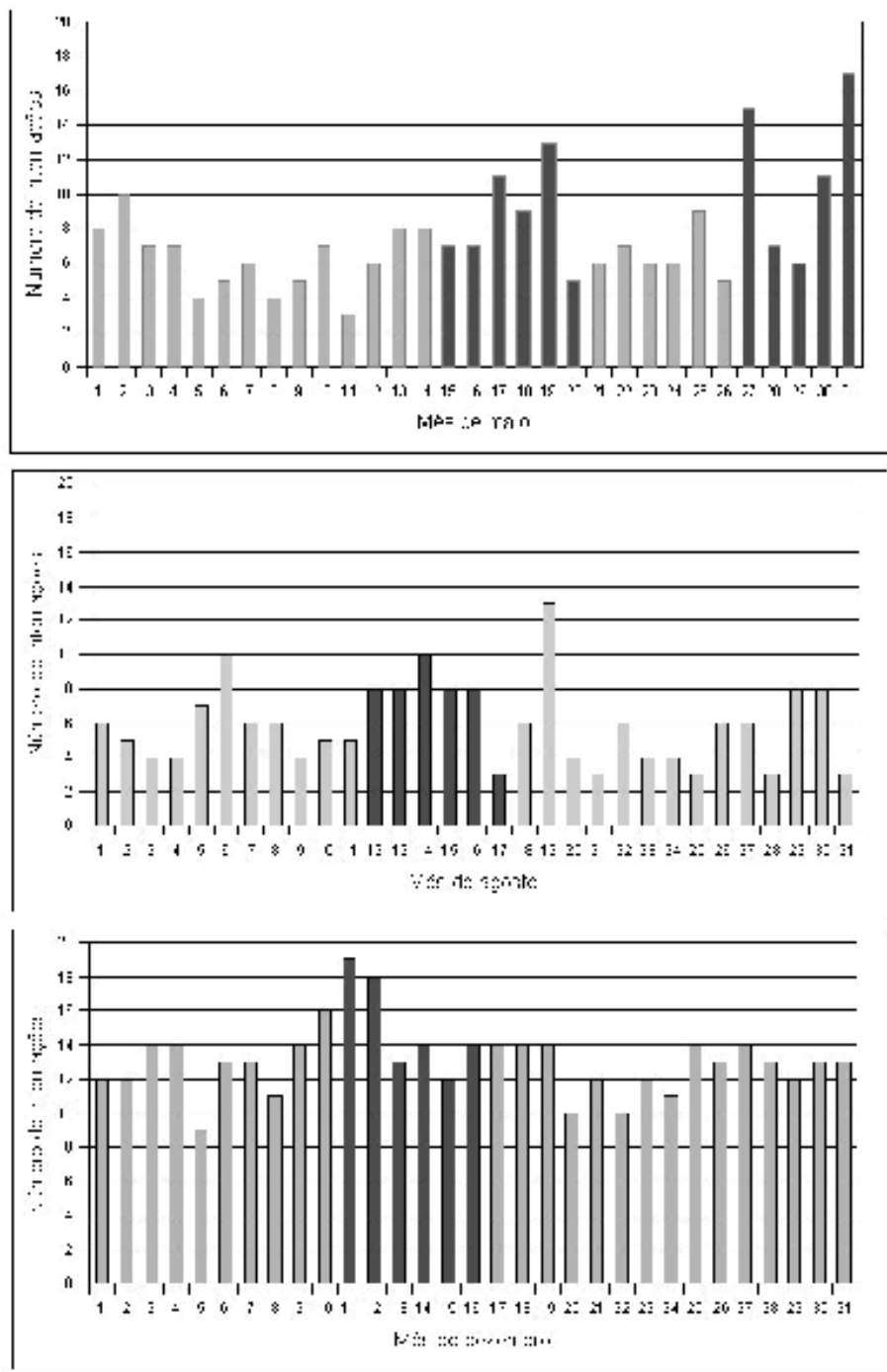

FIGURA 3: Número de internações por dia para os meses de maio, agosto e dezembro, respectivamente, a), b) e c). Cinza claro representa o número de hospitalizações por dia e cinza escuro àqueles relacionados com os casos de maior concentração de poluentes $($ maio e dezembro $=$ PTS; agosto $=$ PTS e $\mathrm{O})_{3}$. 
NÓBREGA, M.R \& KRUSCHE, N. Diagnóstico qualitativo da poluição ...

$\mathrm{O}$ mês de dezembro concentra o maior número de baixas hospitalares por doenças respiratórias em relação aos outros meses do ano de 2002. No dia da máxima concentração, 11 de dezembro, constata-se o maior número de baixas por esse tipo de doença ao longo do referido mês. $\mathrm{O}$ dia posterior à máxima concentração de PTS registra o segundo maior número de internações durante esse período. $\mathrm{O}$ aumento das baixas em dezembro coincide com o período de ocorrência de concentrações elevadas de componentes poluidores e os cinco dias posteriores às mesmas.

Os bairros mais próximos às indústrias registraram, entre os 105 casos de internações por doenças do trato respiratório para o mês em análise, cerca de $43 \%$ desses concentrados no período de máxima concentração de poluentes e dias posteriores. Aproximadamente $48 \%$ das internações de crianças menores que cinco anos durante o mês enfatizado concentraram-se entre os dias 11 a 16 de dezembro de 2002.

\section{Análise das condições meteorológicas para o total de episódios de poluição do ar em 2002}

A análise do comportamento das variáveis meteorológicas para os 27 episódios de poluição de PTS e O3, revela que, em 65\% das ocorrências, a pressão encontrava-se abaixo da normal de pressão, a temperatura encontrava acima da normal de temperatura em $83 \%$ dos episódios, e a umidade relativa encontrava-se abaixo da normal em cerca de $74 \%$ dos dias. Verifica-se, também, que de acordo com o Quadro 4, em cerca de $83 \%$ dos dias, ocorreu nenhuma precipitação ou inferior a $5 \mathrm{~mm}$. Quanto à intensidade da velocidade do vento, verifica-se que as intensidades mais freqüentes encontram-se abaixo de $2,1 \mathrm{~m} / \mathrm{s}$. 
NÓBREGA, M.R \& KRUSCHE, N. Diagnóstico qualitativo da poluição ...

\begin{tabular}{|c|c|}
\hline VARIÁVEIS METEOROLÓGICAS & Ocorrência (\%) \\
\hline Anomalia Negativa de Pressäo & 65 \\
\hline Anomalia Positiva de Temperatura & 83 \\
\hline Anomalia Negativa de Umidade Relativa & 74 \\
\hline Taxa de Precipitação Inferior a $5 \mathrm{~mm}$ & 83 \\
Intensidade da Velocidade do Vento $<2,1 \mathrm{~m} / \mathrm{s}$ & 61
\end{tabular}

QUADRO 3: Comportamento das variáveis meteorológicas no total de episódios de PTS e $\mathrm{O}_{3}$ acima dos padrões de qualidade do ar.

Verificou-se que, em todos os 27 eventos, a média da intensidade do vento não ultrapassou $5 \mathrm{~m} / \mathrm{s}$, caracterizando uma brisa leve, ventos que traduzem condições de calmaria, característica típica que favorece a concentração de componentes poluidores próximos à superfície. Considerando todos os casos de máxima concentração de poluentes para cada estação de monitoramento, verificou-se, que apenas no dia 15 de maio, ocorreram ventos com intensidade superior a $5 \mathrm{~m} / \mathrm{s}$, durante as 12 horas, registrando $8 \mathrm{~m} / \mathrm{s}$.

Durante os cinco episódios de contaminação do ar enfatizados neste estudo, destaca-se a influência da direção do vento no transporte de poluentes, considerando a distribuição das estações de monitoramento da qualidade do ar e as indústrias dentro do espaço urbano do município.

Observa-se, com base na Figura 2, que a ocorrência do episódio do dia 15 de maio, registrada na estação RGPRM, com condições de ventos na direção sudoeste, favorecem o transporte de poluentes oriundos das indústrias localizadas ao sul e sudoeste dentro do município. Essa associação já foi apontada por Bortolini e Krusche (1998), revelando que essa direção de ocorrência de ventos propicia que a pluma emitida pelas indústrias seja direcionada para esta estação. O episódio do dia 12 de agosto, registrado pela estação RGRC, sob direção de ventos que oscilam entre o quadrante sudoeste e noroeste, permite-nos inferir que a presença de ventos de direção sudoeste propicia que as emissões 
NÓBREGA, M.R \& KRUSCHE, N. Diagnóstico qualitativo da poluição ...

das indústrias de fertilizantes possam ser transportadas na direção das estações de monitoramento. Evidencia-se, também, que devido à proximidade das indústrias em relação às estações de monitoramento, a direção do vento pode tornar-se um fator de pequena influência. Os demais casos ocorridos no dia 25 de agosto, 27 de maio e 11 de dezembro, estavam sob condições de ventos com maior freqüência no quadrante norte.

A análise dos cinco dias posteriores aos episódios, constata a mudança da direção do vento de norte para sudoeste, nos eventos registrados nas estações RGCE, RGCO E RGCE (estação móvel), pode ter influenciado para o transporte contínuo da pluma emitida pelas indústrias de fertilizantes nos dias sucessivos a esses casos de contaminação do ar, dada a predominância de ventos inferiores à $5 \mathrm{~m} / \mathrm{s}$. Ressalta-se, ainda, que o registro de poluentes para a estação RGCE, em 25 de agosto, pode estar associado à proximidade das indústrias, sem sofrer influência da direção do vento. O predomínio da direção do vento sudoeste para os dias que sucedem o episódio registrado pela estação RGRC, favorece o transporte de poluentes emitidos pelas indústrias de fertilizantes em direção a esta estação de monitoramento.

A análise dos cinco dias posteriores para o total de episódios revela que, em apenas um caso de máxima concentração de poluentes, a direção do vento permaneceu igual ao dia do episódio, no dia 15 de maio.

A análise do comportamento da temperatura em três eventos de contaminação do ar revela que predomina o decréscimo da mesma em relação aos quatro dias que antecedem os mesmos, pois se constata temperaturas menores no dia do evento. Um dia após o fato, verifica-se que a temperatura decresce em $80 \%$ dos eventos de poluição do ar.

O episódio ocorrido no dia 25 de agosto revelou expressivo aumento da temperatura, considerando o dia que antecede o caso e no dia de ocorrência do mesmo, pois a temperatura registrada no dia desse evento foi elevada em relação à média de agosto, tendo 
NÓBREGA, M.R \& KRUSCHE, N. Diagnóstico qualitativo da poluição ...

este acréscimo da temperatura favorecido a concentração do $\mathrm{O}_{3}$, visto que dias quentes favorecem sua formação.

O comportamento da pressão atmosférica para os quatro dias anteriores ao episódio permite inferir que esta diminui no dia de ocorrência do mesmo, pois os quatro dias anteriores revelam valores de pressão atmosférica superiores ao encontrado no dia do episódio. Um dia após a ocorrência de elevada concentração de agentes poluidores, verificou-se que a pressão atmosférica revela um comportamento ascendente em $60 \%$ dos casos. O que pode ser explicado com base no estudo de Baumbach e Krusche (2005), pelo escoamento de um centro de alta pressão a leste de Rio Grande, proporcionando um decréscimo da pressão.

A análise da variável umidade relativa para os quatro dias anteriores aos episódios, revelou o comportamento crescente desta variável até o dia de ocorrência do mesmo. Após a ocorrência dos casos, mais precisamente no dia que sucede estes, verificou-se o aumento desta variável para $80 \%$ das ocorrências.

A taxa de precipitação revela que apenas dois episódios ocorreram sob a presença de chuva. Após a ocorrência dos casos, verificou-se a presença de chuva nos dias posteriores em três deles em que tinham máxima concentração, como já foram enfatizados nesta pesquisa.

\section{Sistemas de tempo atuante durante os episódios de máxima concentração de poluentes e internações por doenças respiratórias}

O comportamento das variáveis meteorológicas (item 4.2) é justificável dentro da análise do contexto dos sistemas de tempo atuantes na região. A análise dos sistemas meteorológicos atuantes na região durante o período abrangido nesta investigação foram alvo de estudo de outra pesquisa. E, portanto serão apresentados aqui como subsídios para a complementação de uma análise visando a totalidade da apreensão. Nóbrega, Baumbach e Krusche (2005), ao estudarem os sistemas de tempo atuantes na região, para os episódios máximos de concentração de poluentes abordados no 
NÓBREGA, M.R \& KRUSCHE, N. Diagnóstico qualitativo da poluição ...

subitem presente, afirmam que no dia 15 de maio, os campos de baixa pressão e ventos convergentes caracterizam um sistema préfrontal, o qual é confirmado pelo gradiente de temperatura e alta umidade relativa.

Os dias 15 e 27 de maio e 25 de agosto de 2002 são caracterizados por um sistema pré-frontal, marcado pela baixa pressão e convergência dos ventos. Nestes dias, um centro de alta pressão encontra-se a leste da região, conforme observado por Baumbach e Krusche (2005). O episódio do dia 12 de agosto é todo ele marcado por um sistema de alta pressão, gerando um anticiclone sobre a cidade de Rio Grande, o que proporciona ventos fracos na região de estudo. No episódio de 11 de dezembro, um centro de alta pressão encontra-se estacionado a sudeste de Rio Grande, enquanto que um sistema de baixa começa a se aproximar da região. Os ventos são ciclônicos moderados (NOBREGA et al, 2005).

\section{Conclusão}

O estudo do comportamento das internações por doenças respiratórias, no período de 2000 a 2002, revelou que moradores de bairros mais próximos às indústrias são internados com maior constância que moradores de bairros mais distantes. Sendo que as baixas hospitalares são mais freqüentes em crianças menores que cinco anos e durante o inverno, o que sugere situação típica de inversão térmica, favorecendo a concentração de poluentes próximos à superfície.

A associação entre níveis de poluentes, hospitalizações por doenças do trato respiratório e condições meteorológicas, revela o aumento do número de internações durante os cinco dias posteriores à ocorrência de altas concentrações. As condições meteorológicas caracterizam que os eventos de poluição do ar estão associados ao estabelecimento de sistemas de alta pressão, temperaturas altas, ausência de precipitação e ventos fracos. 
NÓBREGA, M.R \& KRUSCHE, N. Diagnóstico qualitativo da poluição ...

A percepção da população riograndina acerca dos efeitos adversos à saúde, provenientes da poluição apontadas pela população quanto às características meteorológicas favoráveis e desfavoráveis à concentração de poluentes próximos à superfície, coincidem com os resultados provenientes das análises meteorológicas realizadas neste estudo.

A população residente dos bairros mais afastados das indústrias sofre menor influência dos efeitos da poluição da atmosférica do que os residentes dos bairros mais próximos às fontes de contaminação do ar. A poluição do ar não se restringe, portanto, apenas aos bairros em que as indústrias estão localizadas.

Os resultados aqui apresentados sugerem que é preciso novos estudos que corroborem com inúmeros estudos nacionais que indicam que apenas os padrões de qualidade estabelecidos pelo CONAMA, hoje não são parâmetros suficientes para garantir um ambiente saudável, no que tange à qualidade do ar.

\section{Agradecimentos}

Ao Conselho Nacional de Desenvolvimento Científico e Tecnológico (CNPq), que financiou o projeto QUARELP (Diagnóstico Qualitativo da Poluição do Ar na Região Estuarina da Lagoa dos Patos), resultando neste artigo. Ao Centro de Processamento de Dados da Associação de Caridade Santa Casa de Misericórdia, ao Setor de Custos do Hospital Universitário, em especial aos funcionários Marcos e Gilmar, respectivamente. À Estação Meteorológica de Rio Grande e à Fundação de Proteção Ambiental Henrique Roessler, por terem fornecido os dados necessários à realização deste trabalho.

\section{Referências bibliográficas}

BAUMBACH, M.O.; KRUSCHE, N. Condições Atmosféricas Favoráveis a Concentração de Poluentes em Rio Grande, RS , Brasil, Durante o Ano de 1994. In: CONGRESSO DE MeteorologiA, 9., 2005, Buenos Aires. Anais do IX 
NÓBREGA, M.R \& KRUSCHE, N. Diagnóstico qualitativo da poluição ...

Congresso de Meteorologia, Buenos Aires: [s.n.], 2005. p. 11.111.6 (Anais..., v. 1)

BELL, M.L. et al. Challenges and Recommendations for the Study of Socioeconomic Factors and Air Pollution Health Effects. Environmental Science and Policy, England, v. 8, p. 525-533, 2005.

BORTOLINI, M.; KRUSCHE, N. Análise da Qualidade do Ar em Rio Grande Durante o Ano de 1997. In: CONGRESSO BRASILEIRO DE METEOROLOGIA, 10., 1998, Brasília. Anais... Brasília: [s.n.], 1998.

BRAGA, A.L.F. Quantificação dos Efeitos da Poluição do Ar sobre a Saúde da População Pediátrica da Cidade de São Paulo e Proposta de Monitorização. 1998. Tese (Doutorado em Medicina) - Faculdade de Medicina, Universidade de São Paulo, 1998.

KRUSCHE, N.; SARAIVA, J.M.B.; REBOITA, M.S. Normais Climatológicas Provisórias de 1991 a 2000 para Rio Grande, RS. Rio Grande: [s.n.], 2002.

MIRLEAN, N.; CASARTELLI, M.R.; GARCIA, M.R.D. Propagação da Poluição Atmosférica por Flúor nas Águas Subterrâneas e Solos de Regiões Próximas às Indústrias de Fertilizantes. Química Nova, São Paulo, v. 25, n. 2, p. 191-195, 2002.

NÓBREGA, M.R.; BAUMBACH, M.O; KRUSCHE, N. Condições Meteorológicas, Níveis de Poluentes e Internações por Doenças Respiratórias em Rio Grande, RS, Durante o ano de 2002. In: SIMPÓSIO BRASILEIRO DE GEOGRAFIA FÍSICA APLICADA, 11., 2005, São Paulo. Anais... São Paulo: [s.n.], 2005. p. 1378-1384 (Anais..., v. 1) 
NÓBREGA, M.R \& KRUSCHE, N. Diagnóstico qualitativo da poluição ...

SALDIVA, P.H. N. et al. Association Between Air Pollution and Mortality due to Respiratory Diseases in Children in São Paulo, Brazil: a preliminary report. Environmental Research, v. 65, p. 218-225, 1994.

SALDIVA, P.H.N. et al. Air Pollution and Mortality in Elderly People: a time-series study in São Paulo, Brazil. Archives of Environmental Health, v. 50, n. 2, p. 159-163, 1995.

SARAIVA, L.B.; KRUSCHE, N. Análise de Situações Atmosféricas Favoráveis à Concentração de Poluentes em Rio Grande, RS, Através de Componentes Principais. CONGRESSO BRASILEIRO DE METEOROLOGIA, 12., 2002, Foz de Iguaçu. Anais... Foz de Iguaçu: [s.n.], 2002. p. 2077-2085

STRAHLER, A.; STRAHLER, A. Introducing Physical Geography. New York: John Wiley and Sons, 1996.

VANZ, A.; MIRLEAN, N.; BAISCH, P. Avaliação de Poluição do Ar por Chumbo Particulado: uma abordagem geoquímica. Química Nova, São Paulo, v. 26, n. 1, p. 25-29, 2003.

Recebido em fevereiro de 2010 Aceito em abril 2010 